\title{
Development of Fertigation Control System Based on Embedded Platform and Self-adaptive Control Strategy
}

\author{
Yin Niu, a , Kanyu Zhang ${ }^{2, b}$ \\ ${ }^{1,2}$ Department of electromechanical engineering and automation, \\ Shanghai University, Shanghai 200072, China; \\ any@agriscience.com.cn, ${ }^{\text {b }}$ kanyu_zhang@agriscience.com.cn
}

Keywords: fertigation, ARM, $\mu$ C/OS-II, self-adaptive, ANN-PID.

\begin{abstract}
In order to improve the performance of fertigation equipment, a fertigation control system based on ARM and $\mu \mathrm{C} / \mathrm{OS}-\mathrm{II}$ is developed. Serial screen is adopted to improve the performance of human-machine interaction, and to decrease the load of MCU. Several tasks related to the control system and fertigation process are designed based on the schedule of $\mu \mathrm{C} / \mathrm{OS}$-II. Furthermore, a self-adaptive control algorithm combined the single neuron with PID is proposed. The testing results of EC and $\mathrm{PH}$ control demonstrate that this method is effective to the fertigation control system.
\end{abstract}

\section{Introduction}

With rapidly development of facility agriculture and fertigation technology in our country, the demand of precise regulation for water and fertilizer is increasing higher. In developed countries of modern agriculture, such as Netherlands and Isreal, high performance fertigation equipments are widely used for management online of water-fertilizer process of crop, but the degree in automatic control field of fertigation in China is relatively low. At present, there are mainly three kinds of fertigation equipments applied in China, including differential pressure mixer, liquamatic injection mixer, and mechanical drive injection mixer ${ }^{[1]}$. Venturi is a kind of liquamatic injector, which was the most widely used because of its simple structure and low-cost. The majority of foreign intelligent fertigation equipments also use venturi as the suction component. However, due to high cost and lacking of after-sale service, the imported equipments are not suitable for domestic promotion. Recent years, some homemade intelligent fertigation equipments have been emerged by absorbing the advantages of foreign equipments. Both are similar in the mechanical structure, yet a large gap in the hardcore of control system, such as the convenience of human-machine interaction, control performance, reliability of system.

Some researches about the fertigation control system have been carried out. Cheng Wang etc. ${ }^{[2]}$ designed an irrigation-fertilization controller by using SCM, which can control the concentration of fertilizer and PH of mixing process. Zhang Qiong etc. ${ }^{[3]}$ developed a modular data acquisition control system by taking ARM7 processor as nucleus and using RS-485 bus as communication mechanism between each module, while $\mu \mathrm{C} / \mathrm{OS}-\mathrm{II}$ operating system was adopted for multi-task scheduling. Guifen Chen etc. ${ }^{[4]}$ combined fuzzy logic and neural network with the irrigation control system to achieve water-saving effect.

In order to improve the integration and reliability of the control system in fertigation equipments, and improve the performance of human-machine interaction, an open-tank mixing fertigation equipment with automatic control system based on embedded platform is developed in this paper. In the embedded platform, STM32, an ARM processor, is used as MCU (micro controller unit), LCD and touch screen is used as HMI (human-machine interface), $\mu \mathrm{C} / \mathrm{OS}-\mathrm{II}$ is used for multi-task scheduling. On the basis of embedded platform, ANN-PID self-adaptive control strategy is proposed to control the water-fertilizer process.

\section{Structure of Fertigation Equipment}

One kind of open-tank mixing fertigation equipment is proposed, of which the mechanical structure is shown in fig.1. A mixing tank is adopted instead of direct mixing in pipe for premixing. This buffer 
container will make the fertilizer/acid solution mixed with irrigation water completely before fertigation process. Float valve is used to maintain the level in mixing tank by the way of hydraulic control, and the low level float is used for pump protection.

Several dosing channels are involved in this equipment. Each one composed of a fertilizer/acid stock tank, a fertilizer/acid filter, a venturi injector and a pulse electromagnetic valve. Venturi injector doses fertilizer/acid solution into mixing tank from stock tank. The rated flow of venturi is determined by the difference between upper pressure gauge and lower pressure gauge, which can be fixed by a pressure-regulating valve. The real flow is determined by the user recipe and is realized by controlling the switching time of the pulse electromagnetic valve.

The system flow, concentration and $\mathrm{PH}$ value of nutrient solution are the most important parameters for crop and soil in the fertigation process. The system flow is measured by flow meter, and the $\mathrm{PH}$ value is measured by $\mathrm{PH}$ sensor. Previous studies have shown that there is a certain connection between the concentration and the conductivity of nutrient solution. Therefore, EC (electrical conductivity) sensor is used for indirect measurement of the concentration of nutrient solution.

In process of traditional configuration of the fertilizer/acid solution in stock tank, all fertilizers in terms of certain recipe should be dissolved and mixed in the stock tank completely, then be pumped into the irrigation pipe network. The amount of fertilizers need in a certain fertigation process should be estimated beforehand to decrease fertilizer waste, and to avoid the antagonistic effect among different fertilizers. On the contrary, in the equipment discussed above, each dosing channel can work independently. Thus, several needed elemental fertilizers can be storage in different tanks, and be dosed into mixing tank only if the fertigation process start, by which preparing for fertilizer/acid solution in stock tank will be simplified.
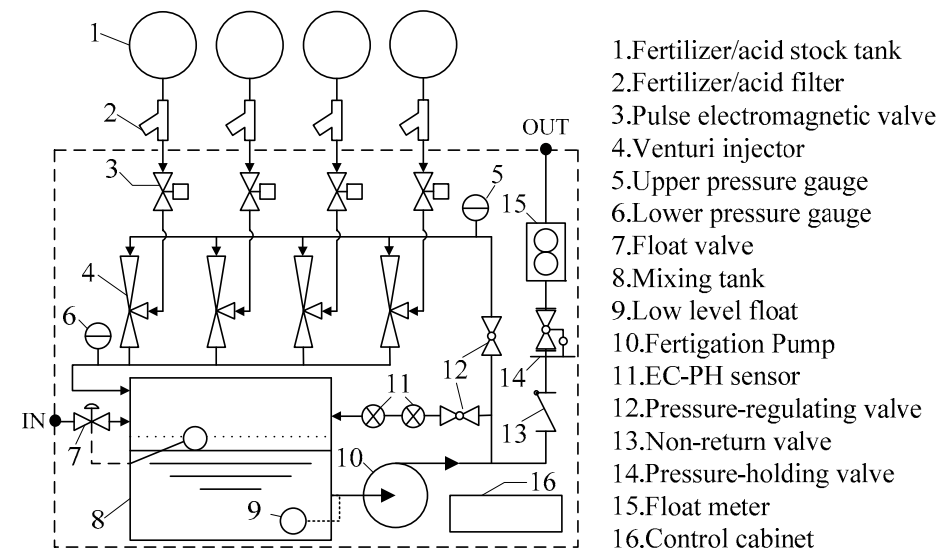

Fig.1 Mechanical structure of open-tank mixing fertigation equipment

In the aspect of control, the concentration and $\mathrm{PH}$ value of mixed nutrient solution are respectively measured by EC and PH sensors on line. The fact dosing quantity and dosing speed are controlled by the pulse electromagnetic valve. A closed control system is composed of controllers, together with data acquisition part and control execution part.

The role of pump is delivering the water-fertilizer mixture into field as well driving the venturi injectors. Pressure-regulating valves could maintain the pressure of pipe.

\section{Embedded Platform Design}

The control cabinet is the core of fertigation equipment, which contains embedded control system and corresponding electrical circuits. The hardware and software designs of the embedded platform will be introduced in this section.

\section{Hardware Platform.}

Stm32F103ZET6 is an ARM microprocessor with 32bit RISC structure based on Cortex-M3, which has $72 \mathrm{MHz}$ working frequency, as integrated $512 \mathrm{~K}$ flash and rich timer resources. That is adopted as MCU in this paper. The architecture of embedded hardware platform is shown in fig.2. There are mainly five function interfaces: analog signal input interface, digital output interface, human-machine interface, storage interface, and network interface. 
Parameters need to be monitored in fertigation control system include EC value and PH value of mixed solution, system flow rate, and water temperature. The signal type of EC sensor, $\mathrm{PH}$ sensor and flow meter is 4-20mA current signal, while the signal type of water temperature is 1-5Vvoltage signal. These data of sensors are processed by TVL2556 that is a 12-bit resolution A/D converter with 11 channels. Processed data are transmitted to the MCU through SPI (Serial Peripheral Interface) bus. Before A/D convert, raw input signals are handled by voltage /current switching, then the operational amplifier LM258 is used for voltage following of input signals. It can improve the interference immunity of analog signal input interface. Moreover, optical coupler (Adum1201) is used between data acquisition module and MCU to improve the safety of system.

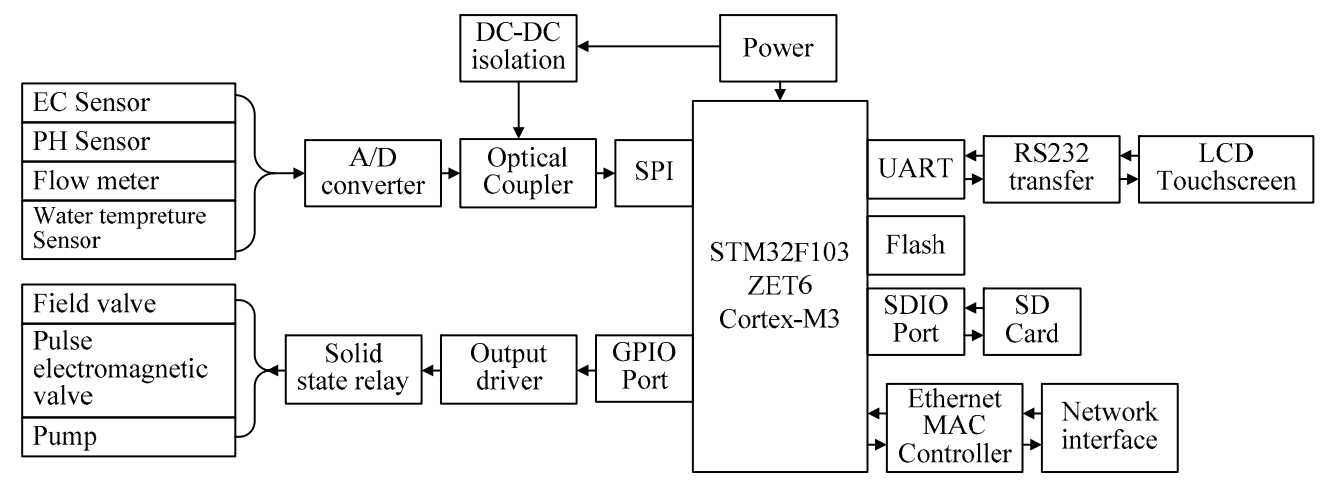

Fig.2 Architecture of embedded hardware platform

The Objects of digital output are valves and pump. Field valves control the fertigation process of each irrigated area, and pump provides power of water system, both are switch signals. Pulse electromagnetic valves control the fact dosing flow. Flow is a continuous variable but there are only two states in pulse electromagnetic valve, so PWM control with periodic switch signal is proposed to solve this problem. There are up to 16 channels to control field valves, up to 6 channels to control pulse electromagnetic valves, and 1 channel to control pump. ULN2003 is used for power amplifying to drive the solid state relay.

The human-machine interface is an important part of system, which consist mainly of parameters display about the fertigation process, irrigation strategy setting, irrigation program setting, recipe setting, system setting and so on. The interactive data contains image data, parameters data for display, and user setting data. For the control system, image data and parameters data for display belong to output data, while user setting data belong to input data. Image data occupy far more operation space than the other data. Along with the increasing size of LCD, the frequency of MCU is needed higher for image data transmission, which will increase the cost of system design. A kind of HMI device with serial port is selected in this system, which integrated with LCD and touch screen. During the system development process, image data about display interface can be storage in HMI device in advance, thus the other data transmit with MCU via RS-232, MAX3232 is used for level-logic switching. The load of MCU is greatly reduced by this design.

The data need to be stored contain system variables and fertigation process data. These data will help users to optimize the management of water and fertilizer. There is an on-chip flash with $512 \mathrm{~K}$ bytes in MCU, using for system variables storage. The fertigation process data are stored in external SD card, including acquisition data of sensors, user setting data, alarm data, by which history data are convenient to be read out.

The network interface is an extended interface, which enable other system connect with this equipment. It realized by DM9000, a fast Ethernet MAC controller, based on the TCP/IP protocol.

In addition, power supply subsystem is also important. Several DC-DC conversion chips are involved. Besides supply suitable power to each chip, a special power supply is derived for isolation of data acquisition channels. 


\section{Software Platform.}

The fertigation control system needs to carry out custom irrigation process in time, to execute periodic fertilizer control, to implement system monitor data acquisition on line, to complete data display and storage task and so on.

The $\mu \mathrm{C} / \mathrm{OS}-\mathrm{II}$ is a kind of RTOS based on preemptive kernel with portability, configurability and scalability. It contains basic functions such as task schedule, task management, time management, memory management, communication and synchronization among tasks. Besides, it is an open source kernel with little space occupation, which can combine with microcontroller without $\mathrm{MMU}^{[6]}$. In this paper, $\mu \mathrm{C} / \mathrm{OS}$-II is used for multi-task scheduling.

\section{(1) Transplantation of $\mu \mathrm{C} / \mathrm{OS}-\mathrm{II}$}

In order to build an OS platform for multi-task design, the initial work is transplanting $\mu \mathrm{C} / \mathrm{OS}-\mathrm{II}$ into the hardware platform. The architecture of embedded software platform is illustrated in fig.3.

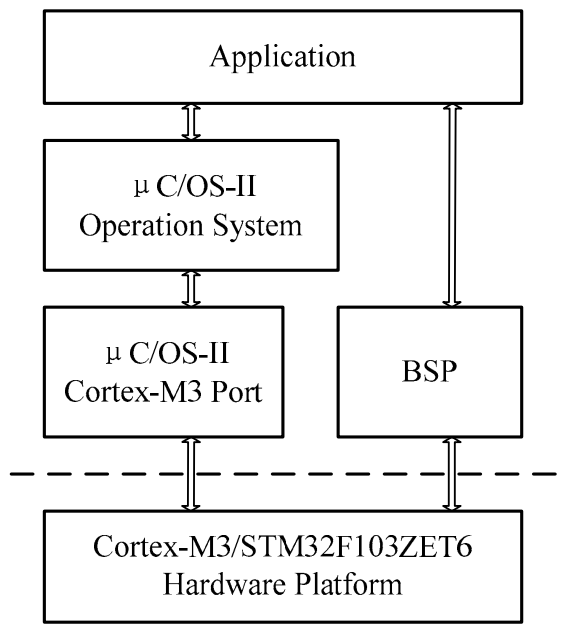

Fig3. Architecture of embedded software platform

The source of $\mu \mathrm{C} / \mathrm{OS}-\mathrm{II}$ can be divided into two parts: general source and port related source. The general source is service to applications. We need to modify the port related source aim at STM32, which involve three files: os_cpu_a.asm, os_cpu.h, os_cpu_c.c. The correct initializing and hardware interrupts handling are two important works of transplantation.

The $\mu \mathrm{C} / \mathrm{OS}-\mathrm{II}$, as with all real-time kernels, needs to disable interrupts in order to access critical sections of code and re-enable interrupts when done. Two macros are defined to disable and enable interrupts. For STM32, a method is selected to implement this process by writing a function that will save the status register of the CPU in a variable and invokes another function to restore the status register from this variable ${ }^{[5]}$. Furthermore, the stack grow direction should be set from high memory to low memory. The function OSTaskStkInit() should be implemented to initialize the stack frame of STM32 for the task being created. Finally, as $\mu \mathrm{C} / \mathrm{OS}-\mathrm{II}$ uses the PendSV handler for context switching and the SysTick handler to process system ticks, PendSV interrupt should be triggered in function OSStartHighRdy().

The BSP(Board Support Package) encapsulates various operations of hardware function interface for application, such as interface initializing functions, read/write functions, send/receive functions, ISR handler functions, output control functions and so on.

Done with descriptions above, the whole embedded platform has been set up. Multi-task about the fertigation control system can be designed based on this platform.

\section{(2) Multi-task Design}

The system acquisition data, run state data and user data are the core of control system. Centre on that several tasks have been set up. The multi-task diagram is shown in fig.4.

Sensor data collecting and processing of each channel are accomplished by data acquisition task, which is an event drive task. A message is sent to mailbox periodically by Timer_ISR, notifying that data acquisition task need to run. When data processing is done, system acquisition data would be created and meanwhile would be sent to message queue for HMI display task. 
Two tasks relevant to HMI are respectively HMI display task and HMI key handle task. The HMI display task takes real data from message queue, and sending it to HMI through RS-232 for real data and curve refreshing. With triggered by usart_isr, user data would be received and be analyzed by the HMI key handle task, then current user configuration would be updated.

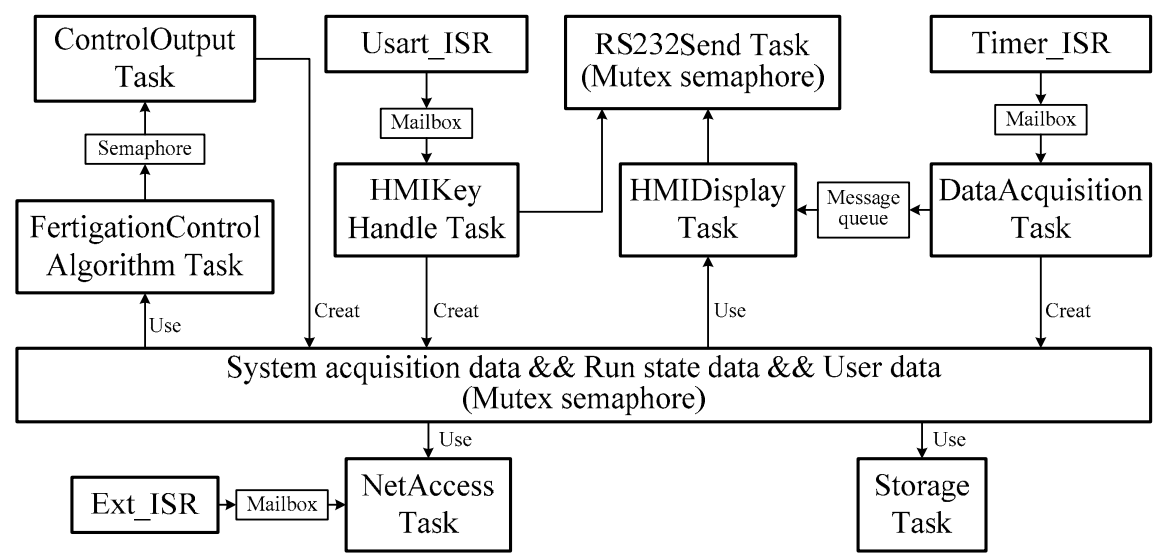

Fig.4 Block diagram of task schedule

Fertigation control algorithm task is a periodic task that composed of fertilizer ratio control, fertilizer concentration control, fertilizer PH control and irrigation strategy control. The control decision in terms of irrigation setting and recipe setting of user is made by this task. A semaphore will be set once a new control decision is created. The output control task executes control task with receiving the semaphore. During the control process, the output control task will continuously detect the system state, and create run state data.

Storage task is also a periodic task. When the SD card is ready, all data about process will be saved in a text file.

Net access task allows remote monitoring from website. When receiving a HTTP request, a message for triggering the net access task will be sent to mail box by ext_isr, and request data will be sent back in HTTP protocol.

The operation system clock is set to $10 \mathrm{~ms}$. Static priority is assigned for tasks. The lowest priority is set to 30 , and the former 4 priorities have been reserved. The shared data resources are maintained by a mutex semaphore for accessing safety, of which the priority is higher than any task.

\section{ANN-PID Control Strategy}

The EC and PH control of fertilizer are important to crop and soil in fertigation process. Nonlinear, strong coupling, uncertainty are characteristics of the control object. PID control strategy was widely used in many process control system with advantage of high efficiency, but it is only suitable to linear control system. It has been proved that the artificial neural network (ANN) has strong learning capability for adapting a nonlinear control system. A self-adaptive control method combined with single neuron (three inputs, one output) and incremental PID algorithm is proposed in this paper. The structure of this method is shown in fig. $5^{[7]}$.

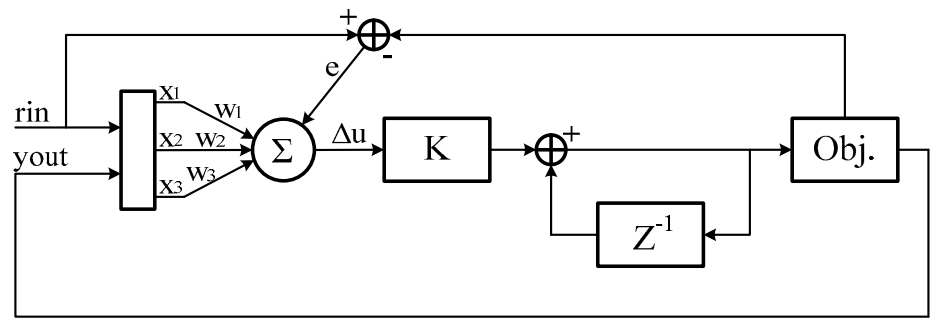

Fig.5 Structure of ANN-PID control system

The control law is expressed as follows: 


$$
u(k)=u(k-1)+K \cdot \sum_{i=1}^{3} w(k) x_{i}(k)
$$

Where $K$ is the system gain, and inputs of neuron $x_{i}(k)$ are respectively error, error differential, error integral between system output and expected output:

$$
\begin{aligned}
& x_{1}(k)=e(k) \\
& x_{2}(k)=e(k)-e(k-1) \\
& x_{3}(k)=\Delta^{2} e(k)=e(k)-2 e(k-1)+e(k-2)
\end{aligned}
$$

The learning method of weights expressed as follows:

$$
\begin{gathered}
w(k)=w_{i}(k) / \sum_{i=1}^{3}\left|w_{i}(k)\right| \\
w_{1}(k)=w_{1}(k-1)+\eta_{I} \cdot e(k) \cdot u(k-1) \cdot(e(k)+\Delta e(k)) \\
w_{2}(k)=w_{2}(k-1)+\eta_{P} \cdot e(k) \cdot u(k-1) \cdot(e(k)+\Delta e(k)) \\
w_{3}(k)=w_{3}(k-1)+\eta_{D} \cdot e(k) \cdot u(k-1) \cdot(e(k)+\Delta e(k))
\end{gathered}
$$

Where $w(k)$ is normalized weight vector, while $\eta_{I}, \eta_{P}, \eta_{D}$ are learning rate. $e(k)$ is teacher signal.

\section{Results and Discussion}

Two testing processes for both EC control and PH control with the self-adaptive control algorithm are implemented. The control algorithms are included in the fertigation control algorithm task. For EC control, the control cycle is $6 \mathrm{~s}$, the expect value are respectively $0.8 \mathrm{~ms} / \mathrm{cm}$ and $1.2 \mathrm{~ms} / \mathrm{cm}$, while for $\mathrm{PH}$ control, the control cycle is $3 \mathrm{~s}$, the expect value are respectively 6.5 and 6.0. The results of ANN-PID control method for EC and PH control process is demonstrated in fig.6.

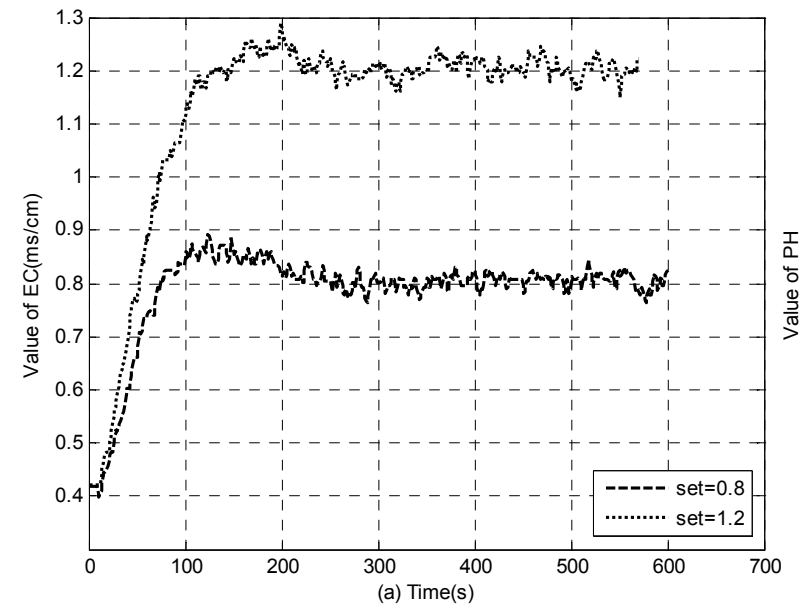

Fig.6 (a) EC control process (b) PH control process

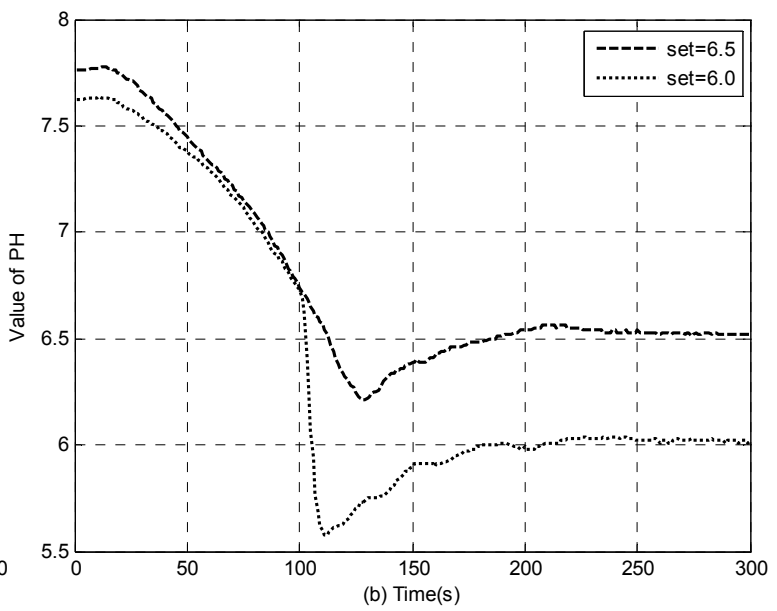

It is shown that the $\mathrm{EC}$ and $\mathrm{PH}$ value of fertilizer can achieve and steady at the expect value in different situations by method discussed above. In EC control process, there is a little fluctuation in system output because of disturb. In PH control process, due to the accuracy of sensor is 0.1 , there is steady state error approximately 0.05 in system output. More studies about nonlinear system control will be carried out in future research.

\section{Conclusion}

A fertigation control system that uses STM32 with ARM Cortex-M3 and RTOS of $\mu \mathrm{C} / \mathrm{OS}-\mathrm{II}$ based on a kind of open-tank mixing fertigation equipment was developed. More specially, the embedded platform has been established by STM32 and $\mu \mathrm{C} / \mathrm{OS}-\mathrm{II}$ successfully. Serial screen not only improved the performance of human-machine interaction, but decreased the load of MCU. Software development with modularity was simplified that was benefit from $\mu \mathrm{C} / \mathrm{OS}-\mathrm{II}$. Based on the embedded 
platform, a self-adaptive control strategy combined single neuron with incremental PID is proposed, and meanwhile, testing for EC and $\mathrm{PH}$ process in different situations are applied. The results verified that this method is effective to the fertigation control system.

\section{Acknowledgement}

This research was supported by the Science and the Technology Committee of Shanghai Project "Research on the key technologies of energy-saving in the full closed greenhouse" (No. 10DZ2212300). The author would like to thank the company of Bangbo Modern Agriscience for providing test condition especially.

\section{References}

[1] Li Kai, Mao Hanping, Li Baijun. Analysis on Control Performance of Various Pesticide or Fertilizer Mixers[J]. Transactions of the Chinese

Society for Agricultural Machinery, 2003, 34(1): 50-53.

[2] Cheng Wang, Chunjiang Zhao, Xin Zhang, Xiaojun Qiao, Yinghua He. Research and Exploitation of Precise Irrigation-Fertilization Controller[C].2007 Second IEEE Conference on Industrial Electronics and Applications, 2007:172-175.

[3] Zhang Qiong, Zhao Chunjiang, Wang Cheng, Xu Zhilong. Data acquisition control system based on ARM processor for automatic fertigation[J]. Transactions of the CSAE, 2008, 24(Supp.2): 44-49.

[4] Guifen Chen, Lisong Yue. Research of Irrigation Control System Based on Fuzzy Neural Network[C]. 2011 International Conference on Mechatronic Science, Electric Engineering and Computer, Jilin,2011:209-212.

[5] Micri $\mu m$. $\mu \mathrm{C} / \mathrm{OS}-\mathrm{II}$ and ARM Cortex-M3 Processors Application Note[DB/OL]. http://micrium.com/download/.

[6] Jean J. Labrosse. $\mu$ C/OS-II The Real-Time Kernel[M]. U.S.A., 2003.

[7] Jianhua Yang, Wei Lu, and Wenqi Liu. PID Controller Based on the Artificial Neural Network[J]. Springer-Verlag Berlin Heidelberg 2004:144-149 